\title{
Efficacy and patient satisfaction with autoadjusting CPAP with variable expiratory pressure vs standard CPAP: a two-night randomized crossover trial
}

\author{
A. T. Mulgrew $\cdot$ R. Cheema $\cdot$ J. Fleetham $\cdot$ C. F. Ryan $\cdot$ \\ N. T. Ayas
}

Published online: 20 October 2006

(C) Springer-Verlag 2006

\begin{abstract}
Expiratory pressure relief (C-Flex) technology monitors the patient's airflow during expiration and reduces the pressure in response to the patient. Increased comfort levels associated with C-Flex therapy have potential to improve patient adherence to therapy. The purpose of this study was to assess the combination of autoadjusting CPAP (APAP) and C-Flex in terms of (1) treatment efficacy, and (2) patient preference when compared to standard CPAP. Fifteen patients who had previously undergone formal CPAP titration polysomnography were treated with either one night of the APAP with C-Flex or one night of conventional CPAP, in a crossover trial. Patient satisfaction levels were recorded using visual analog scales (VAS) on the morning after the study. Mean patient age was $50 \pm$ 12 years, body mass index (BMI) was $36 \pm 6 \mathrm{~kg} / \mathrm{m}^{2}$, baseline AHI was $53 \pm 31$ events $/ \mathrm{h}$, and CPAP Pressure was $11 \pm 2 \mathrm{~cm} / \mathrm{H}_{2} \mathrm{O}$. APAP with C-Flex was as effective as
\end{abstract}

A. T. Mulgrew $\cdot$ J. Fleetham $\cdot$ C. F. Ryan $\cdot$ N. T. Ayas

Respiratory Division, Department of Medicine,

University of British Columbia,

Vancouver, British Columbia, Canada

\section{N. T. Ayas}

Centre Clinical Epidemiology and Evaluation,

Vancouver Coastal Health Research Institute,

Vancouver, British Columbia, Canada

A. T. Mulgrew $\cdot$ R. Cheema $\cdot$ J. Fleetham $\cdot$ C. F. Ryan $\cdot$ N. T. Ayas Sleep Disorders Program, Vancouver Acute Hospitals,

Vancouver, British Columbia, Canada

Present address:

N. T. Ayas $(\triangle)$

2775 Heather Street,

Vancouver, British Columbia V5Z 3J5, Canada

e-mail: najib.ayas@vch.ca
CPAP, with no differences detected in sleep latency $(17 \pm 5$ vs $12.3 \pm 3 \mathrm{~min}, p=0.4$ ), or respiratory indices (AHI of $4.2 \pm 2$ vs $2.4 \pm 0.7$ events $/ \mathrm{h}, p=0.1$ ). VAS scores (scale $0-10$ ) indicated a trend towards increased patient satisfaction while using APAP with C-Flex (7.9 vs 7.2, $p=0.07$ ). 10 patients expressed a preference for APAP with C-Flex (VAS, 0 to 10) over standard CPAP (total positive score of 68 , mean score of $4.8 \pm 4.3$ ). One patient expressed no preference. Four patients expressed a preference for CPAP (total positive score of 13, mean score of $0.9 \pm 1.9$ ) (APAP with C-Flex vs standard CPAP, $p<0.01$ paired $t$ test). APAP with C-Flex eliminates sleep disordered breathing as effectively as standard CPAP. Patients indicated a preference for APAP with C-Flex suggesting a possible advantage in terms of patient adherence for this mode of treatment.

Keywords CPAP.C-Flex · Auto-CPAP. Patient preference

\section{Introduction}

Continuous positive airway pressure (CPAP) is the most commonly prescribed treatment for patients with obstructive sleep apnea (OSA). CPAP is effective in reducing sleep fragmentation, resolving nocturnal desaturation, and improving daytime sleepiness [1]; moreover, CPAP is a costeffective use of healthcare resources, and may reduce risks of motor vehicle crashes and the development of cardiovascular morbidity [2-5].

The efficacy of CPAP is compromised by relatively poor compliance. A variety of measures have been shown to improve compliance with CPAP; these include aggressive follow-up of patients prescribed CPAP, group education, 
heated humidification, and mask adjustments [6-11]. Modifications in the algorithm, by which positive pressure is delivered, have been attempted to improve comfort and compliance with therapy.

A typical complaint by those using conventional CPAP is that it is difficult to exhale against positive pressure. Therefore, reducing pressure during exhalation may improve tolerance with therapy. C-Flex (Respironics, Murrysville, PA, USA) is a novel algorithm designed to provide pressure relief during expiration, while maintaining optimal pneumatic splinting for effective therapy.

Another modification is the development of autoadjusting (APAP) devices. In contrast to conventional CPAP, APAP devices modify applied pressure in response to patient needs. APAP devices, through varied mechanisms, are able to detect obstructive events and change pressure accordingly to relieve the obstruction. This results in a reduction in mean applied CPAP pressure [12], and may be useful in patients who have difficulty tolerating CPAP. Furthermore, APAP may be useful for ambulatory titration of CPAP pressure in patients in whom in-laboratory therapy titration is either delayed or impossible [13-15]. The newest iteration of the REMStar Auto employs both the REMStar Auto CPAP algorithm and the C-Flex dynamic expiratory pressure relief.

This is the first study to evaluate the REMStar Auto with C-Flex (RSA C-Flex) as a therapy for patients with OSA. The major purpose of the study was to assess the ability of RSA C-Flex to effectively treat the sleep apnea events. Satisfaction and preference from the patients' perspective, and the number of required interactions per night by technicians were also determined.

\section{Materials and methods}

\section{Study subjects}

Consecutive, recently diagnosed, adult patients ( $\geq 18$ years of age) with moderate to severe OSA [apnea hypopnea index (AHI) $>15$ events/h of sleep as determined by PSG] [16] were asked to participate in our study. All subjects had a titration PSG that determined the effective pressure (i.e., AHI $<5$ events/h during all sleep stages and positions). All subjects were using conventional CPAP at home on a regular basis with an average nightly usage $>4 \mathrm{~h}$, confirmed by objective evaluation of CPAP machines. Exclusion criteria included patients who were medically unstable, using oxygen, had surgery of the upper airway or nose within the previous 90 days, or who could not provide informed consent.

The study was approved by the Ethics Board at the University of British Columbia.
Procedures and techniques

Sleep laboratory rooms were configured with a device that could deliver either conventional CPAP or RSA C-Flex. The study occurred on two separate nights, within 2 weeks of each other. For the first night, patients were randomized to either conventional CPAP at the previously determined effective pressure or RSA C-Flex. The patient was not aware of which of the therapies they would be receiving. However, the technician, by necessity, was aware of the therapy choice. On the second experimental night, subjects received the alternative therapy (crossover design).

For each night, all subjects were instrumented for polysomnography and PAP titration according to practice parameters published by the American Sleep Disorders Association Standards of Practice Committee [17]. Chest and abdominal excursion was measured using respiratory impedence plethysmography (Respitrace; Ambulatory Monitoring Equipment, Ardsley, New York, USA). Oxygen saturation was recorded using a pulse oximeter (Model N100; Nellcor, Hayward, California, USA). Airflow was measured directly from the CPAP machine. Respiratory events were defined by standard criteria [16]. Obstructive apneas were defined as absence of airflow for greater than $10 \mathrm{~s}$. Obstructive hypopneas as a $50 \%$ decrease in airflow, or a clear but lesser decrease in airflow if coupled with either a desaturation of $>3 \%$ or an arousal in the context of ongoing respiratory effort. Central apneas were defined as absence of airflow and respiratory effort for greater than $10 \mathrm{~s}$. Central hypopneas as a $50 \%$ decrease in airflow with concomitant reduction in respiratory effort, or a clear but lesser decrease in airflow if coupled with either a desaturation of $>3 \%$ or an arousal. Arousals were counted per hour of total sleep time. Sleep efficiency index was defined as total sleep time/total time in bed.

Satisfaction with devices was assessed on the morning after the study (see attached surveys, Appendix 1). All subjects completed a visual analog satisfaction (VAS) questionnaire. VAS scores are useful in assessing subjective patient experience and have been used for this purpose in a previous crossover trial comparing conventional CPAP and APAP [18]. Patients scored satisfaction (0-10) with different aspects of their experience from the previous night. Questions included: (1) How well did you sleep last night? (2) How rested do you feel this morning? (3) How often did you wake during the night? (4) How uncomfortable was your CPAP pressure? (5) How difficult was it to get to sleep? (6) Overall, how satisfied were you with your quality of sleep last night? Mean score was calculated for all 15 patients on both nights.

Preference for device was assessed on the morning after the second experimental night. Subjects were asked "In comparison to the first night of the study, please state a 
preference for the CPAP machine set-up". Subjects were asked to place a mark on a line to indicate the degree of preference for the first night $(0-10)$ or the second night $(0-10)$. Mean score was calculated for both nights, with a score of less than 0 indicating preference for the other night.

The technicians recorded any required interactions with the subject, during the night.

\section{Conventional CPAP without C-Flex}

CPAP pressure was set to the therapy pressure determined during a previous titration study. A 20-min ramp was used with the initial pressure being set as $66 \%$ of the previously titrated pressure.

\section{RSA with C-Flex}

The REMStar Auto algorithm (Respironics, Murrysville, PA, USA) is a proactive, flow-based algorithm. The basic algorithm evaluates inspiratory flow, and determines impending or actual flow limitation. This flow evaluation occurs in concert with a program of pressure adjustments designed to evaluate the critical pressures (at which airways are susceptible to collapse) and resolve therapy pressures slightly above these critical pressures. The patient is protected from "break-through" events with a full complement of intelligent responses to airflow events and snoring. C-Flex technology monitors the patient's airflow during expiration and reduces the pressure in response to the patient. The relief pressure may vary on a breath-to-breath basis, depending on the actual patient airflow (Fig. 1). The device can be preset to deliver varying degrees of C-Flex pressure relief (settings of 1-2-3).

RSA C-Flex was set at a minimum pressure of $66 \%$ of previously titrated therapy pressure and a maximum pressure of $20 \mathrm{~cm} \mathrm{H}_{2} \mathrm{O}$. Patients were arbitrarily set to a $\mathrm{C}$-Flex setting of 3 (maximum pressure relief). If the patient commented about any sensation of "breathlessness" due to the pressure relief, comfort settings were adjusted to 2 or 1 by the night technician.

\section{Statistical analysis}

Baseline characteristics were described using means and standard deviations for continuous variables. Differences between the CPAP and RSA C-Flex arms were compared using paired $t$ tests. Two-tailed tests were used to assess significance (at the 0.05 level).

\section{Results}

Fifteen patients were included in the trial. Baseline characteristics included (mean \pm SD) age $50 \pm 12$ years, BMI $36 \pm$ $6 \mathrm{~kg} / \mathrm{m}^{2}$. Overall, the patients had substantial sleep apnea with a mean baseline AHI of $53 \pm 31$ with a mean CPAP pressure of $11 \pm 2 \mathrm{~cm} \mathrm{H}_{2} 0$.

Results for the RSA C-Flex vs standard CPAP are shown in Table 1. Overall, RSA C-Flex was as effective as standard CPAP in relieving upper airway obstruction with a mean AHI of 4.2 vs $2.4 / \mathrm{h}$ ( $p=0.1$, Fig. 2). One patient had central apneas during the RSA C-Flex night (index 10.2/h). When these central events were excluded, the mean AHI during the RSA C-Flex night decreased to 3.2 events/h (Fig. 2). Furthermore, 12 out of 15 patients had an AHI of less than five on the APAP night, which is similar to the proportion on standard CPAP therapy (13/15). Measures of sleep efficiency were similar between the groups. Although patients had more slow-wave sleep during the RSAC night, this was not statistically significant.

Patient satisfaction levels were measured using a visual analog scale (Fig. 3). In general, VAS scores tended to be higher on the RSA C-Flex night, although none of the scales individually demonstrated a significant difference.
Fig. 1 Pressure relief is shown on a breath by breath basis for the three standard gain settings. Pressure relief is dependant on patient flow according to the formulae $-p_{\text {delivered }}=p_{\text {base }}-$ $p_{\text {relief }} \cdot p_{\text {relief }}=($ flow $*$ gain $)$

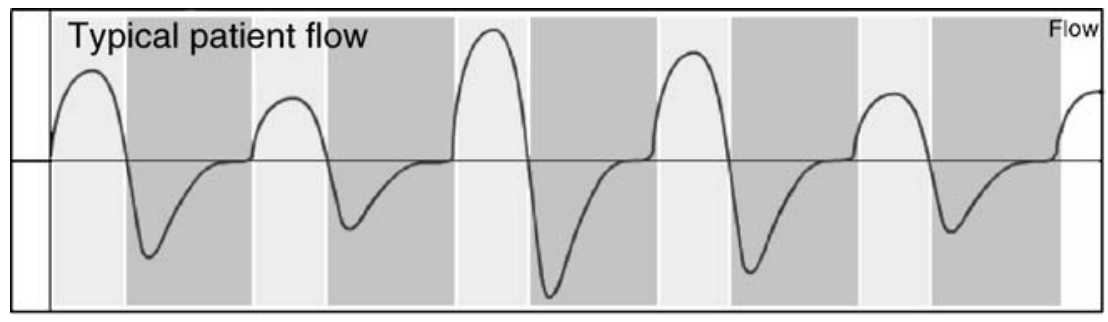

Inspiration

Expiration

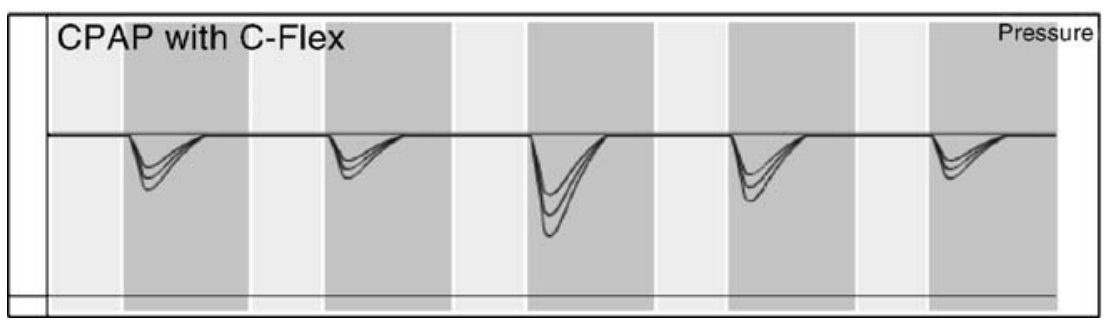

Inspiration

Expiration 
Table 1 Outcomes with RSA C-Flex vs standard CPAP

\begin{tabular}{llll}
\hline & RSA C-Flex & CPAP & $P$ value \\
\hline AHI (events/h) & $4.2 \pm$ & $2.4 \pm 0.7$ & 0.1 \\
Sleep latency (min) & $17.0 \pm 5$ & $12.3 \pm 3$ & 0.4 \\
Sleep efficiency (\%) & $82 \pm 2$ & $83 \pm 2$ & 0.6 \\
WASO (min) & $67 \pm 14$ & $65 \pm 12$ & 0.9 \\
REM \% & $25 \pm 2$ & $24 \pm 2$ & 0.7 \\
Stage 3/4 (\%) & $4 \pm 1.5$ & $1.6 \pm 0.5$ & 0.13 \\
Mean oxyhemoglobin & $97.3 \pm 0.3$ & $97.2 \pm 0.3$ & 0.93 \\
saturation (\%) & & & \\
PLMI (events/h) & $2.8 \pm 1.0$ & $3.8 \pm 1.5$ & 0.59 \\
\hline
\end{tabular}

Values given as mean \pm SEM

AHI Apnea hypopnea index, WASO wake after sleep onset, PLMI periodic limb movement index

However, when Mean VAS scores were calculated for individual patients and compared using a paired $t$ test, a trend towards an increased score was noted in the C-Flex group ( 7.9 vs $7.2, p=0.07$, Fig. 4 a).

In addition, patients were asked to rate their preference for the treatment mode. Ten patients expressed a preference for the RSAC night with the sum of positive scores favoring the RSAC of 68 (mean 4.8 \pm 4.3 ). Four patients expressed a preference for CPAP with the sum of positive scores favoring CPAP of 13 (mean $0.9 \pm 1.9$ ). One patient expressed no preference. A significant difference was found in preference between these two arms (paired $t$ test, $p<0.01$ ) (Fig. 4b).

Sleep technicians recorded the number of interactions with each patient during the night. The majority of these interactions were to adjust recording leads, and a minority of occasions to adjust mask position. Technician interactions did not differ between nights (1.4 vs 2.3 per night, $p=0.14$ ).

\section{Discussion}

In our study, RSA C-Flex was as effective as standard CPAP in treating sleep-disordered breathing. There were no differences in sleep-related breathing or architecture variables between the arms. However, the RSA C-Flex arm was associated with a trend in improvement in patient satisfaction as assessed by VAS scores. Patients preferred RSA C-Flex over standard CPAP.

To our knowledge, only one other published study has looked at the use of C-Flex in OSA. In a nonrandomized study of 89 patients, Aloia et al. [19] examined treatment adherence in patients with OSA assigned to either standard CPAP or C-Flex therapy. Clinical outcomes were measured at 3 months and although functional outcomes associated with sleep did not differ, adherence was significantly greater in the C-Flex group ( 4.8 vs $3.1 \mathrm{~h} /$ night, $p<0.01$ ). The crossover design of our trial using random treatment order guaranteed patient matching while allowing us to assess patient preference. Our study is not directly comparable to that of Aloia et al. as they examined long-term compliance. However, our results are consistent in that our two-night study demonstrated a preference of the patient for RSA C-Flex.

Our findings are consistent with other studies of autoadjusting CPAP therapy. A metaanalysis of nine randomized controlled trials demonstrated that APAP is as effective as standard CPAP in treating sleep apnea (in terms of reducing apnea hypopnea index) [12]. Although this metaanalysis did not demonstrate a significant improvement in compliance with APAP, other studies have suggested that APAP may be preferred by patients, especially if their baseline CPAP pressures are elevated [20]. To control this, we used 20-min ramps during CPAP nights in an attempt to match initial pressures with the RSA C-Flex night.
Saturation

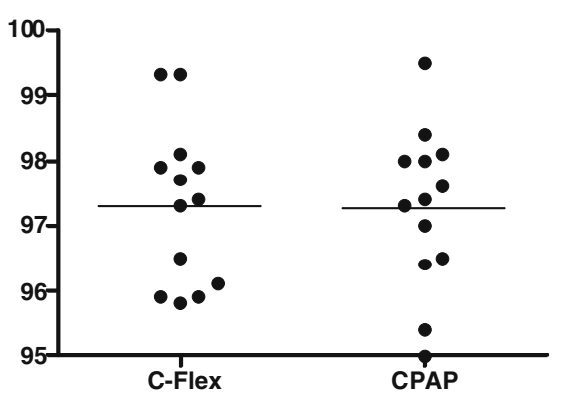

Fig. 2 Mean oxygen saturation and AHI were compared between the treatments. Mean AHI was 4.5 in the C-Flex arm, as compared to 2.5 in the standard CPAP arm $(p=0.1)$. When central events were
AHI

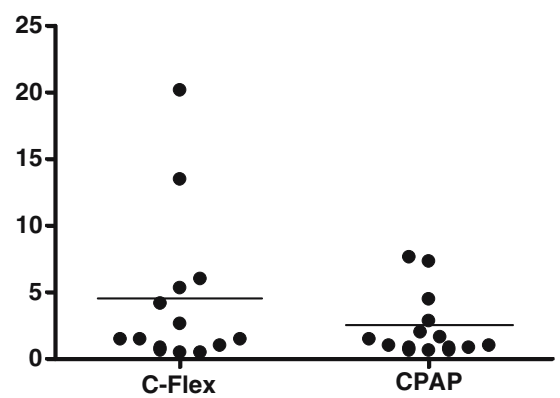

excluded, this decreased to 3.5 in the C-Flex arm compared to 2.5 in the CPAP arm $(p=0.12)$ 

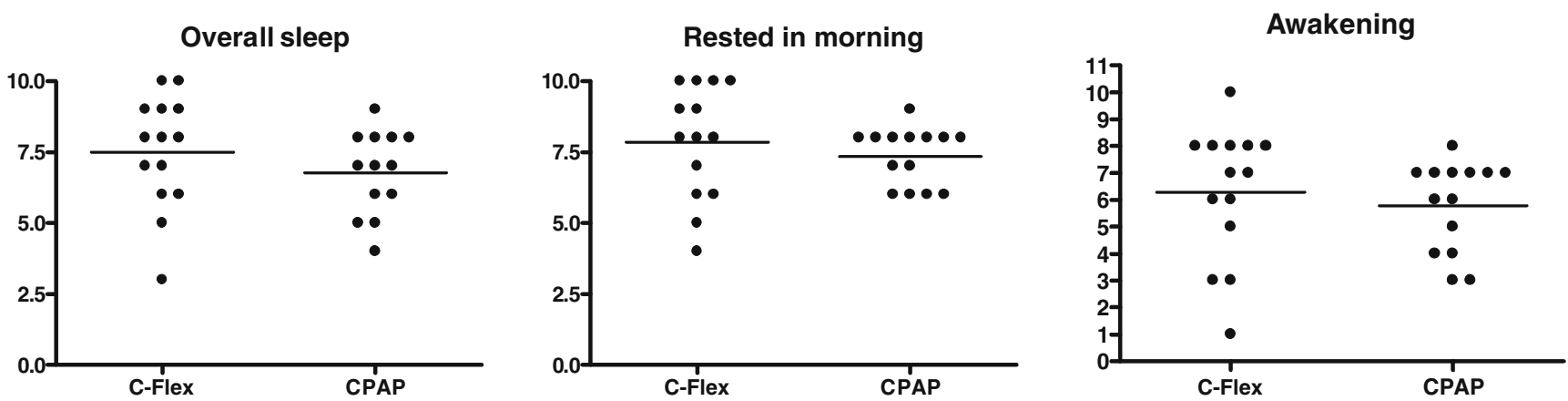

\section{Comfortable with pressure}
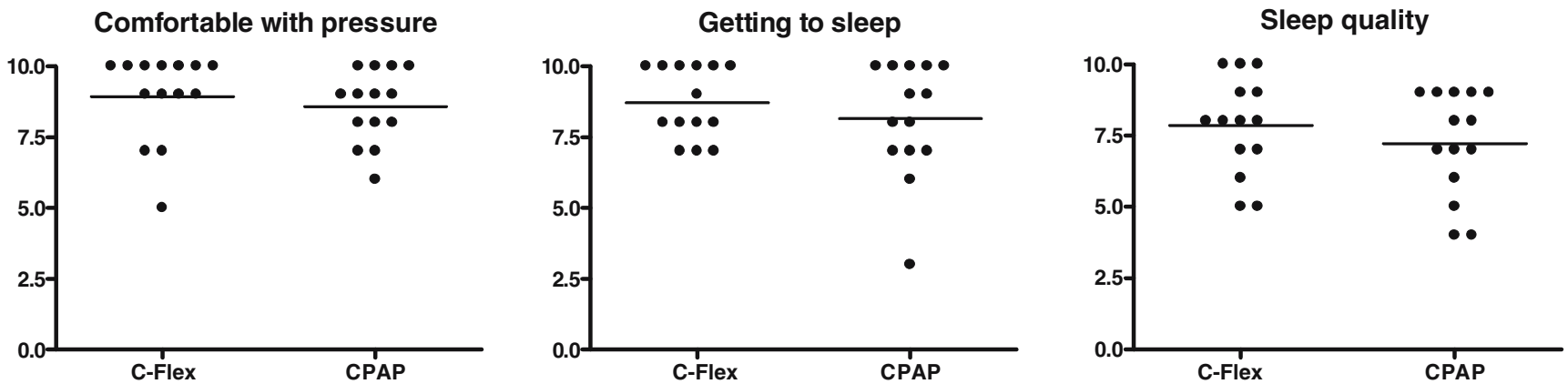

Fig. 3 Visual analog scales were used to determine patient comfort levels. Scales ranged from 0-10 and were administered on the morning post sleep study. Although VAS scores were higher on the C-Flex night, differences were not significant using a paired $t$ test ( $p>0.1$ for all outcomes)

In this two-night study, we showed that RSA C-Flex is as effective as standard CPAP in reducing AHI. We noted that one patient with a treatment AHI of $>20$ had a high proportion of central sleep apnea. Review of this patient's diagnostic study revealed a very high baseline AHI $(>100 / \mathrm{h})$ with the presence of central apneas (index $>20 / \mathrm{h}$ ). As such we feel that residual central apneas in this patient reflect baseline disease rather than being caused by RSA C-Flex. However, the possibility that the RSA C-Flex may predispose to central events in a subgroup of patients cannot be excluded.

Patients preferred RSA C-Flex over standard CPAP, and may have found it more comfortable. As such, we speculate that RSA C-Flex may improve compliance if used as chronic therapy. Furthermore, one of the major determinants of longterm compliance with CPAP is the early experience with the therapy. For many patients, in-laboratory titration represents the first exposure of any duration to CPAP therapy (Appendix 2). Any positive impact on this initial experience has the potential to improve compliance in the longer term. Use of RSA C-flex may thus be useful for initial titration of CPAP in the laboratory. For similar reasons, RSA C-flex may be useful in patients who are being titrated at home with APAP. The impact of RSA C-Flex on long-term compliance with chronic use, as "salvage" therapy of patients intolerant of standard CPAP, or its utility as part of a laboratory/ ambulatory CPAP titration need to be studied in larger clinical trials.

We acknowledge that there are a number of limitations to our study. First, although the patient was not told of the nightly treatment assignment, the technician was not blinded. For our study, it was not possible for the technician to be unaware of the treatment. Second, we have studied a relatively small number of patients. Third, we have only studied patients for two nights in the laboratory and as such, we cannot extrapolate our study to chronic treatment. We have not tested CPAP adherence
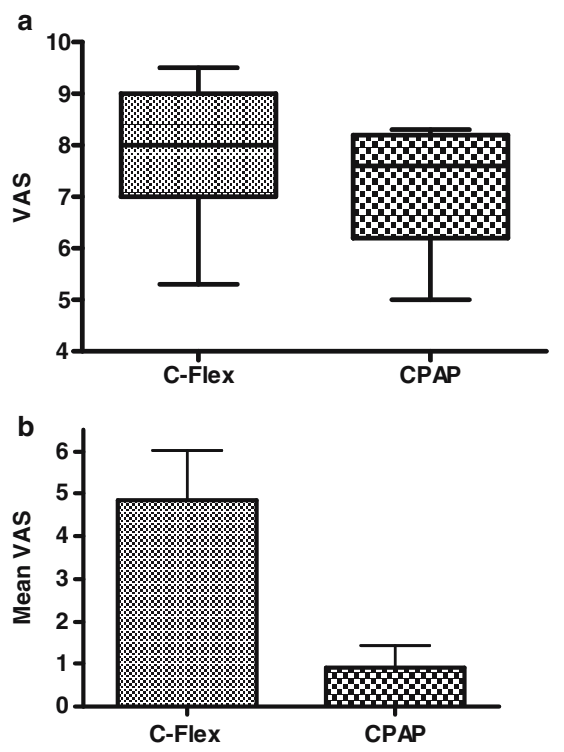

Fig. 4 a Mean VAS scores were calculated for individual patients and compared using a paired $t$ test. A trend towards increased score was noted in the C-Flex group (7.9 vs 7.2, $p=0.07)$. b Patient scored their preference using VAS scores (1-10). A significant preference was noted for C-Flex $(p<0.01)$ 
in this study; our results merely suggest a reasonable hypothesis for future study - that use of the RSA C-Flex may have a favorable effect on compliance. Finally, this study included patients with predominately severe disease who were compliant with CPAP. We may not be able to extrapolate our results to patients with less severe sleep apnea, or to patients with other concomitant respiratory diseases (such as COPD).

\section{Conclusion}

In this small short-term study, both RSA C-Flex and standard CPAP (with pressure defined by an in-laboratory CPAP titration) were effective in treating sleep apnea. Most patients preferred RSA C-flex. Future studies with this technology are warranted to assess its effects on long-term compliance and its potential as rescue therapy for those initially noncompliant with standard CPAP therapy.

Acknowledgement This study was supported by a grant from Respironics. All data were collected and analyzed by the investigators. The manuscript was written by the investigators.

\section{Appendix 1}

REMStar Auto with C-Flex-in-laboratory performance vs conventionally titrated CPAP therapy: performance and preferences

Patient questionnaire

Name Date

Night 1

Thank you for completing the first night of our study. Please take a moment to answer the following questions

1. How well did you sleep last night? Place a mark on the line.

Scale:

0 10

Slept terribly

Slept extremely well

2. How rested do you fell this morning? Place a mark on the line.

Scale:

0 10

Not rested at all Extremely rested

3. How often did you wake during the night? Place a mark on the line.

Scale:

0 10

Woke very often

Didn't wake

4. How uncomfortable was your CPAP pressure? Place a mark on the line.

Scale:

0 10

Extremely uncomfortable

Not uncomfortable
5. How difficult was it to get to sleep. Place a mark on the line.

Scale:

0 10

Extremely difficult

Not difficult

6. Overall, how satisfied were you with your quality of sleep last night. Place a mark on the line.

Scale:

0

Extremely dissatisfied Extremely satisfied

Thank you for completing this survey.

\section{Appendix 2}

REMStar Auto with C-Flex-in-laboratory performance vs conventionally titrated CPAP therapy: performance and preferences

Patient questionnaire

Night 2

Name

Date

Thank you for completing the second night of our study. Please take a moment to answer the following questions

1. How well did you sleep last night? Place a mark on the line.

Scale:

0

Slept terribly

Slept extremely well

2. How rested do you feel this morning? Place a mark on the line.

Scale:

0 10

Not rested at all Extremely rested

3. How often did you wake during the night? Place a mark on the line.

Scale:

0 10

Woke very often Didn't wake

4. How uncomfortable was your CPAP pressure? Place a mark on the line.

Scale:

0 10

Extremely uncomfortable Not uncomfortable

5. How difficult was it to get to sleep. Place a mark on the line.

Scale:

0 10

Extremely difficult Not difficult

6. Overall, how satisfied were you with your quality of sleep last night. Place a mark on the line.

Scale:

0

Extremely dissatisfied

Extremely satisfied 
7. In comparison to the first night of the study please state a preference for the CPAP machine set-up. Place a mark on the line.

Scale:

10 0 10

Preferred first night No preference Preferred second night Thank you for completing this survey.

\section{References}

1. Patel SR, White DP, Malhotra A et al (2003) Continuous positive airway pressure therapy for treating sleepiness in a diverse population with obstructive sleep apnea: results of a metaanalysis. Arch Intern Med 163:565-571

2. Hassaballa HA, Tulaimat A, Herdegen JJ et al (2005) The effect of continuous positive airway pressure on glucose control in diabetic patients with severe obstructive sleep apnea. Sleep Breath 9:176-180

3. Marin JM, Carrizo SJ, Vicente E et al (2005) Long-term cardiovascular outcomes in men with obstructive sleep apnoeahypopnoea with or without treatment with continuous positive airway pressure: an observational study. Lancet 365:1046-1053

4. Ayas NT, FitzGerald JM, Fleetham JA et al (2006) Costeffectiveness of continuous positive airway pressure therapy for moderate to severe obstructive sleep apnea/hypopnea. Arch Intern Med 166:977-984

5. George CF (2001) Reduction in motor vehicle collisions following treatment of sleep apnoea with nasal CPAP. Thorax 56:508-512

6. Taylor Y, Eliasson A, Andrada T et al (2006) The role of telemedicine in CPAP compliance for patients with obstructive sleep apnea syndrome. Sleep Breath (in press)

7. Chervin RD, Theut S, Bassetti C et al (1997) Compliance with nasal CPAP can be improved by simple interventions. Sleep 20:284-289

8. Hoy CJ, Vennelle M, Kingshott RN et al (1999) Can intensive support improve continuous positive airway pressure use in patients with the sleep apnea/hypopnea syndrome? Am J Respir Crit Care Med 159:1096-1100
9. Likar LL, Panciera TM, Erickson AD et al (1997) Group education sessions and compliance with nasal CPAP therapy. Chest 111:1273-1277

10. Massie CA, Hart RW, Peralez K et al (1999) Effects of humidification on nasal symptoms and compliance in sleep apnea patients using continuous positive airway pressure. Chest 116:403-408

11. Mortimore IL, Whittle AT, Douglas NJ (1998) Comparison of nose and face mask CPAP therapy for sleep apnoea. Thorax 53:290-292

12. Ayas NT, Patel SR, Malhotra A et al (2004) Auto-titrating versus standard continuous positive airway pressure for the treatment of obstructive sleep apnea: results of a meta-analysis. Sleep 27: 249-253

13. West J, Stradling (2006) Comparison of three ways to determine and deliver pressure during nasal CPAP therapy for obstructive sleep apnoea. Thorax (in press)

14. Teschler H, Berthon-Jones M, Thompson AB et al (1996) Automated continuous positive airway pressure titration for obstructive sleep apnea syndrome. Am J Respir Crit Care Med 154:734-740

15. Masa JF, Jimenez A, Duran J et al (2004) Alternative methods of titrating continuous positive airway pressure: a large multicenter study. Am J Respir Crit Care Med 170:1218-1224

16. American Academy of Sleep Medicine Task Force (1999) Sleeprelated breathing disorders in adults: recommendations for syndrome definition and measurement techniques in clinical research. The Report of the American Academy of Sleep Medicine Task Force. Sleep 22:667-689

17. American Sleep Disorders Association (1997) Practice parameters for the indications for polysomnography and related procedures. Polysomnography Task Force, American Sleep Disorders Association Standards of Practice Committee. Sleep 20:406-422

18. Hukins C (2004) Comparative study of autotitrating and fixedpressure CPAP in the home: a randomized, single-blind crossover trial. Sleep 27:1512-1517

19. Aloia MS, Stanchina M, Arnedt JT et al (2005) Treatment adherence and outcomes in flexible vs standard continuous positive airway pressure therapy. Chest 127:2085-2093

20. Massie CA, McArdle N, Hart RW et al (2003) Comparison between automatic and fixed positive airway pressure therapy in the home. Am J Respir Crit Care Med 167:20-23 